\title{
Development and assessment offluorinated grapheme nanoparticles modified dental adhesives
}

\author{
Rafal M. Maryoosh ${ }^{1}$, Abdulla M. Al-Shamaa \\ 1B.D.S., M.Sc. (Conservative Dentistry), Ministry of Health/Al-Rusafa Health Department, Baghdad, Iraq, 2 Assist. \\ Professor, B.D.S., M.Sc., Ph.D. (Conservative Dentistry),Department of Conservative, College of Dentistry, \\ University of Baghdad, Baghdad, Iraq
}

\begin{abstract}
Background: The success of adhesion to the tooth structure plays an important role in the dental restoration, which can effectively avoid the terrible clinical problems during the bonding process, including the secondary caries and the loading of adhesive compositions with fillers and nanoparticles with antibacterial properties has led to a significant reinforcement effect of the adhesive.
\end{abstract}

Objective: The current study is designed to investigate the effects of addition of $2 \%$ of fluorinated graphene on the adhesive-dentin hybrid layer for two types of commercially available universal adhesive, guided using the etch and rinse technique as a bonding procedure.

Method: Forty extracted premolar teeth will be divided into four groups of ten teeth. Group I: the nonincorporated (Prime \& Bond Universal) bonding agent (Control group). Group II:using the 2\% FG nanoparticles incorporated (Prime \& Bond Universal) bonding agent. Group III: non-incorporated (All Bond Universal) bonding agent (Control group). Group IV: using the incorporated (All Bond Universal) bonding agent. The degree of conversion was also measured using Fourier transform infrared spectroscopy (FTIR). The antibacterial activity of the adhesives was evaluated using agar diffusion test against the following bacteria: Streptococcus mutans and Lactobacillus salivarius obtained from saliva.

Results: A significantly greater antibacterial activity was obtained with adhesives containing a $2 \%$ fluorinated grap hene nanoparticles than other groups $(P<.01)$. Degree of conversion of tested adhesives was not change significantly after addition of $2 \%$ of fluorinated grap hene nanoparticles.

Conclusion: The antibacterial effect of adhesives that incorporated with $2 \%$ of fluorinated graphene nanoparticles was higher than the non-incorporated etch- and rinse adhesive systems. The degree of conversion of adhesives was not significantly different.

Keywords: Adhesive systems; Antibacterial activity; Degree of conversion; Etch \& Rinse adhesive technique.

\section{Introduction}

Success in adhesive dentistry means long-lasting restorations. Notwithstanding, the loss of adhesion or

Corresponding Author:

Rafal M. Maryoosh

B.D.S., M.Sc. (Conservative Dentistry), Ministry of Health/Al-Rusafa Health Department, Baghdad, Iraq e-mail: rafalmohammed1984@gmail.com retention of a resin composite restoration is a frequent problem observed by dental practitioners, especially due to bond strength degradation ${ }^{(12)}$. The loading of adhesive compositions with fillers and nanoparticles has led to a significant reinforcement effect of the adhesive (3. 4 5).Fluorinated graphene (FG), an up-rising member in the family of graphene derivatives, is a kind of onemolecule-thick material ${ }^{(6)}$. Graphene has been reported to be highly cytotoxic for bacteria and can thus serve as an antibacterialmaterial, so we could speculate that as a 
member of graphene family, FG may also have similar effect $^{(7)}$. In previous studies, the antibacterial function of graphene is believed to be caused by both physical and chemical effects ${ }^{(8)}$.

\section{Materials and Method}

Preparation of modified universal adhesives: Two commercial universal adhesive agents; Prime \& Bond Universal adhesive (Dentsply, Tusla dental specialties, USA (and All- Bond Universal adhesive (Bisco Inc, USA) wereselected in this study. The fluorinated graphene nanoparticles (FGN) (Hyper Chem Co., chaina, Let. No. Q19011701) was used as filler particles and it was submittedto the silanizationprocess to improve the adhesion interface between the filler nanoparticles and the adhesive matrix so that the FGN was treated with3-metacryloxypropyltrimethoxy (MPS) silane coupling agent using a procedure described by Deb et al. in $1996 \square \mathbf{1 2} \square$.

Apercentage of $2 \mathrm{w} \%$ of FGN was chosen from a pilot study out of 4 different percentages (1, 2, 3, 4 w. \%) because this constration didn't adversely affect the viscosity, color stability and flowability of the adhesives $\square^{\mathbf{1 3}} \square$. Then the FGN was dispersed in $6 \mathrm{ml}$ of each adhesive by ultrasonication for $1 \mathrm{~h}$ to obtaining ahomogenous mixture.
Degree of conversion test: For FTIR spectroscopic analysis, equal droplet amount of each adhesive resin was placed on a transparent poly-ethylene film. With a gentle steam of air, the solvents were evaporated for 30 seconds and then covered with a second film and pressed softly to form a thin layer of the adhesive. The "sandwich" was placed into the sample holder of FTIR spectrometer (Equinox 55, Bruker, Germany) (Fig. 3 ), and the absorbance peaks of the unpolymerized adhesives were recorded by transmission mode at a resolution of $4 \mathrm{~cm}-1$, with scans in the range of 400 $4000 \mathrm{~cm}-1 \square \mathbf{1} \square \square$. The adhesives were then light-cured with an LED Bluephase (IvoclarVivadent, Lichtenstein) light-curing unit with a light intensity of $600 \mathrm{~mW} / \mathrm{cm} 2$ for 40 seconds and the absorptions were recorded for the cured adhesive specimens. The DC\% was calculated from the ratio of absorbance intensities for aliphatic $\mathrm{C}=\mathrm{C}$ (peak at $1638 \mathrm{~cm}-1$ ) and the internal reference of aromatic carbon-carbon double bonds (peak at $1608 \mathrm{~cm}-$ 1) were recorded before and after curing the specimens, according to the following equation $\square \mathbf{1} \square \square$ :

$$
\text { DC }=\frac{1-\left(1635 \mathrm{~cm}^{-1} \div 1608 \mathrm{~cm}^{-1}\right) \text { cured }}{\left(1635 \mathrm{~cm}^{-1} \div 1608 \mathrm{~cm}^{-1}\right) \text { uncured }} \times 100 \%
$$

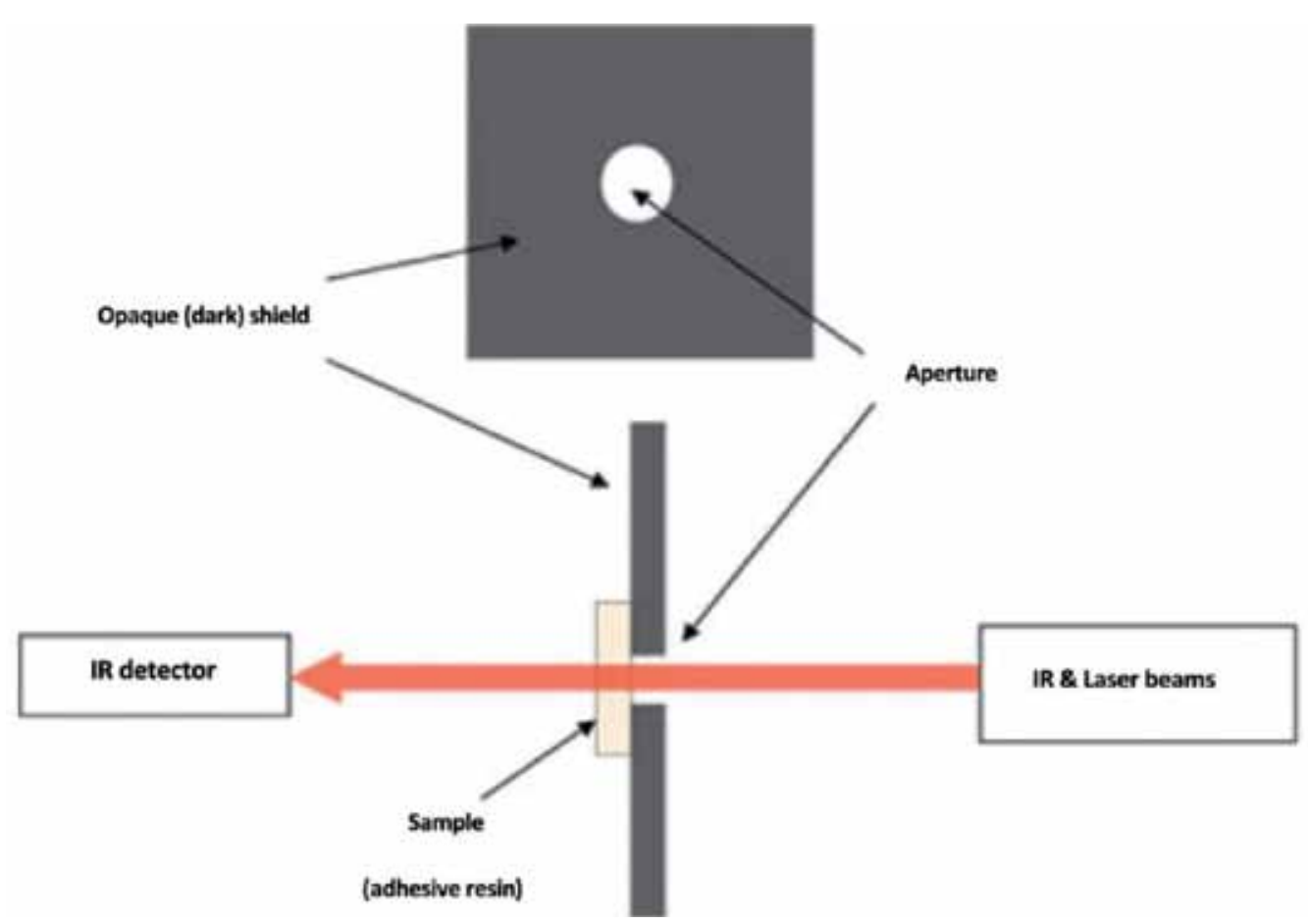

Fig. 3. Set-up for measuring degree of conversion of adhesive resins by Fourier transforms infrared (IR) spectroscopy $\square \mathbf{1} \square \square$. 
Microbiological Study: The microbiological study was conducted in the Department of Microbiology in the Medical Center in Baghdad, to evaluate and test the antibacterial activity of incorporated adhesives and was compared to the control non-incorporated adhesives against selected microorganisms which was Streptococcus mutan sand Lactobacilli, since these are the main microorganisms associated with the development and progression of carious lesion (Primary and secondary caries).

Adhesive specimenwells with an inner diameter of $8 \mathrm{~mm}$ and a depth of $1.0 \mathrm{~mm}$ was prepared. Total forty wells wereprepared, ten wells for each group, 5 wells of each group wereused for each microorganism. The medium that used for antibacterial activity test will be Mueller-Hinton agar for Streptococcus mutans and MRS broth for Lactobacilli (19).

The selective medium for the cultivation of Streptococcus mutans was Mitis Salivarius Bacitracin Agar and for Lactobacilli was MRS Agar $\square^{\mathbf{1}} \square \square$.

A. Culture Medias:

1. Mitis Salivarius Bacitracin Agar (MSB): Mitis salivarius bacitracin (MSB) agar is the selective medium for the cultivation of MutansStreptococci $\square \mathbf{1} \square \square$.

2. MRS Agar (Lactobacillus Selection Agar): MRS Agar was a semi-defined, partially selective medium for the isolation and enumeration of lactobacilli from foods and from intestinal, vaginal, and dental flora $\square \mathbf{1} \square \square$.

\section{B. Isolation of microorganisms:}

The microorganisms were cultured directly from swabs that were taken directly from mouth of volunteer patients. The swab will be rolled over a small area of the surface at the edge on MSB agar and MRS Agar; then was streaked for isolation from this inoculated area.
For MSB agar, the plates was incubated anaerobically utilizing a gas pack for 48 hours at $37{ }^{\circ} \mathrm{C}$ then aerobically for 24 hours at room temperature, while for MRS Agar, the plates was incubated anaerobically for three days at $37^{\circ} \mathrm{C}^{(15)}$

C. Measurement of the inhibition zone: After 24 hours of incubation, the MRS agar plates was removed from the incubator and was examined for the inhibition zone around each well in ( $\mathrm{mm}$ ) after 48 hours of incubation using a ruler around each disc. The measurement of the inhibition zone was done by two microbiologists separately to get the best and reliable standardized results for this test $\square \mathbf{1} \square \square$.

Statistical Analysis: Statistical analysis was done by Statistical Package for Social Sciences (SPSS) version 24. Results were calculated as mean \pm standard error of means (SEM).Comparison among groups was done by using a one-way Analysis of variance (ANOVA). The statistically significant differences were considered when $\mathrm{P}<0.05$.

\section{Results}

Degree of conversion (DC\%): Table 2 and figure 2 showed that there were non-significant differences $(P>0.05)$ in degree of conversion of incorporated All Bond Universal adhesive agent with $2 \%$ fluorinated graphene (Group II) compared to the corresponding degree of conversion of the non- incorporated All Bond Universal adhesive agent (Group I). Mean \pm SEM of degree of conversion of (Group I) \& (Group II) were respectively, $\quad 85.9200 \pm .27479 \mathrm{vs} . \quad 86.2600 \pm .27536$. Similarly, table 2 and figure 2 there were non-significant differences $(P>0.05)$ in degree of conversion of the incorporated Prim \& Bond Universal adhesive agent with $2 \%$ of fluorinated graphene (Group IV) compared to the non-incorporated Prim \& Bond Universal adhesive agent (Group III). Mean \pm SEM of degree of conversion of (Group III) \& (Group IV)were respectively,74.880 $0 \pm .21124$ vs. $75.9200 \pm .18547$.

Table 2: The mean of Degree of conversion (DC) \%in the four experimental groups

\begin{tabular}{|l|c|}
\hline Groups & $\begin{array}{c}\text { Degree of conversion (DC)\% } \\
\text { Mean } \pm \text { SEM }\end{array}$ \\
\hline Group I: The non-incorporated (Prime \& Bond Universal) bonding agent (Control group). & $85.9200 \pm .27479^{\mathrm{a}}$ \\
\hline Group II: The incorporated (Prime \& Bond Universal) bonding agent with 2\% fluorinated graphene & $86.2600 \pm .27536^{\mathrm{b}}$ \\
\hline Group III: The non-incorporated (All Bond Universal) bonding agent (Control group). & $74.8800 \pm .21124^{\mathrm{a}}$ \\
\hline Group IV: the incorporated (All Bond Universal) bonding agent with 2\% fluorinated graphene & $75.9200 \pm .18547^{\mathrm{b}}$ \\
\hline
\end{tabular}




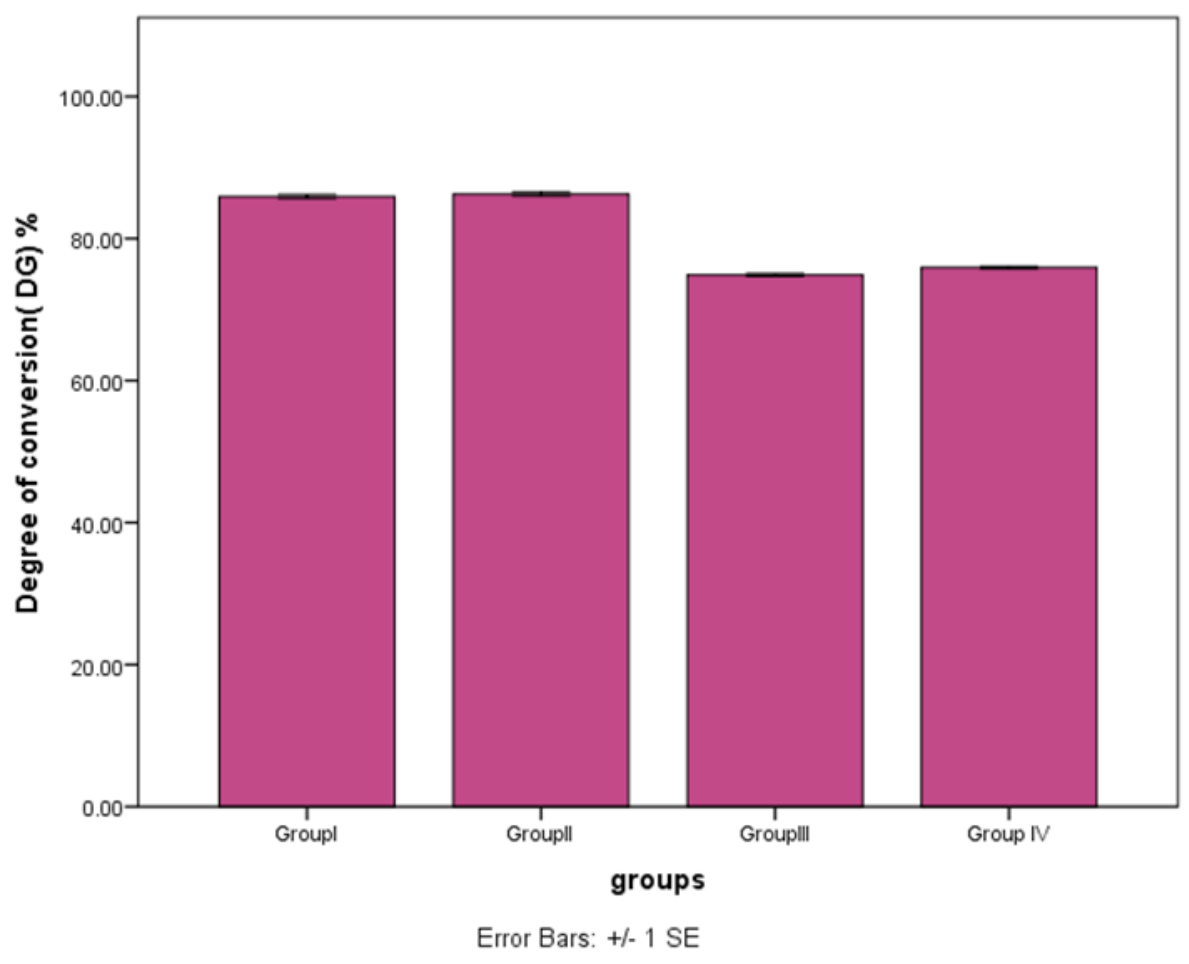

Figure 1. Bar chart showing the mean of Degree of conversion (DC)\% in the four experimental groups

Antibacterial test: The mean and standard error of mean values of the inhibition zone for the Streptococcus Mutans microorganism results for tested adhesive groups are represented in tables (3). The results of this study showed that Group IV: the incorporated (All Bond Universal) bonding agent with $2 \%$ fluorinated graphene exhibited high percentage of reduction of the Streptococcus Mutans microorganisms among the tested adhesive groups as shown in figure (3). Furthermore, ANOVA test shows that there is a highly significant difference in SBS mean group II (the incorporated All Bond universal $+\mathbf{2 \%}$ fluorinated graphene) in compression with group I (the non-incorporated All Bond universal bonding agent $)(\mathbf{p}<.01)$ and there is a highly significant difference in SBS mean group IV (the incorporated Prim \& Bond universal $+2 \%$ fluorinated graphene) in compression with group III (the non- incorporated Prim \& Bond universal bonding agent $)(p<.01)$.

Table 3: The mean of diameter of inhibition in the four experimental groups

\begin{tabular}{|c|c|c|}
\hline Groups & $\mathbf{N}$ & $\begin{array}{c}\text { Diameters of } \\
\text { Inhibition Zone } \\
\text { Mean } \pm \text { SEM }\end{array}$ \\
\hline Group I: The non-incorporated (Prime \& Bond Universal) bonding agent (Control group). & 10 & $0.000 \pm .0000$ \\
\hline Group II: The incorporated (Prime \& Bond Universal) bonding agent with $2 \%$ fluorinated graphene & 10 & $21.700 * \pm 0.232$ \\
\hline Group III: The non-incorporated (All Bond Universal) bonding agent (Control group). & 10 & $0.000 \pm .0000$ \\
\hline Group IV: The incorporated (All Bond Universal) bonding agent with $2 \%$ fluorinated graphene & 10 & $21.8000 \pm 0.133 \#$ \\
\hline
\end{tabular}




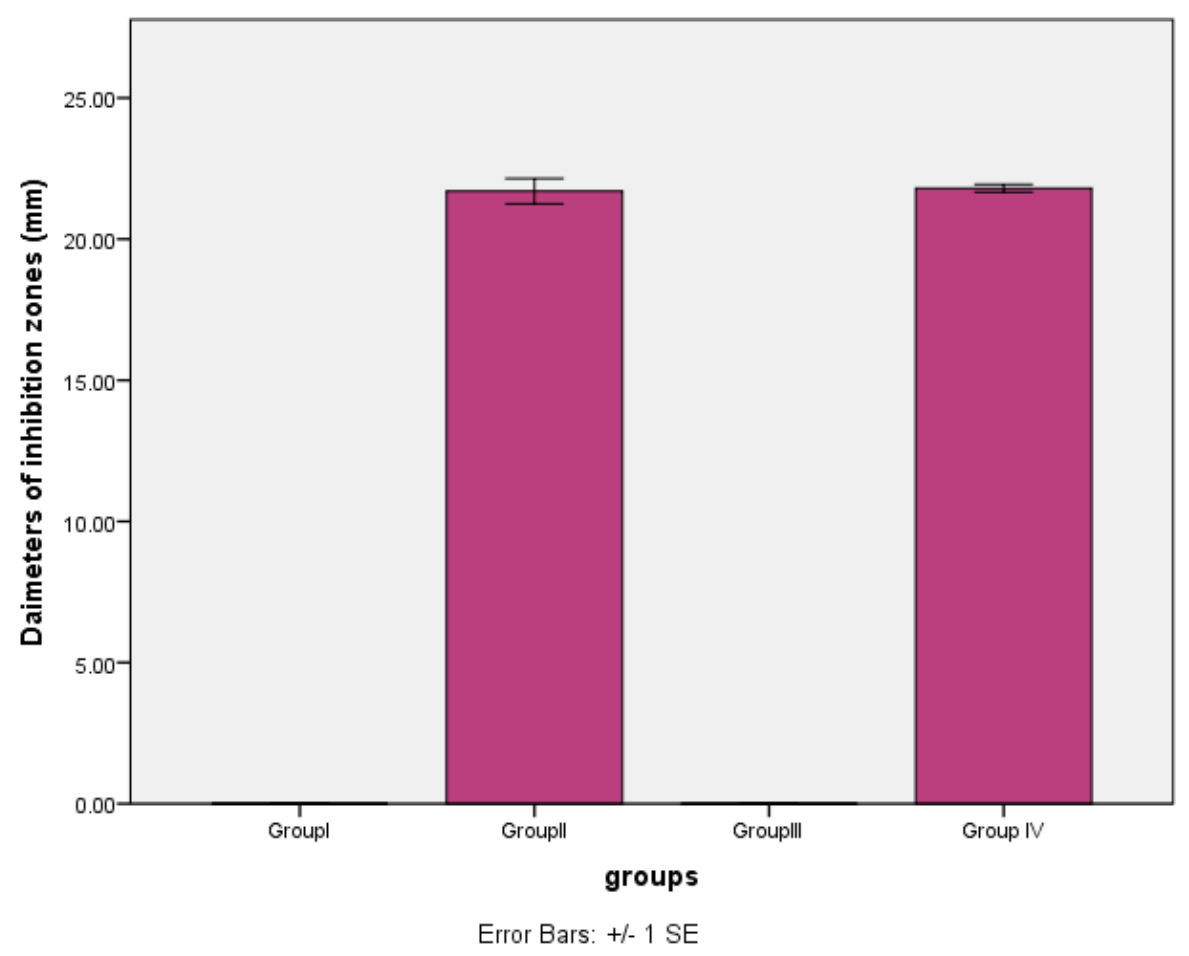

Figure 3. Bar chart showing the mean of Diameters of inhibition zones in the four experimental groups.

\section{Discussion}

Durable and high bond strength to dentin is the goal of all the restorative materials and procedures. Clinical failure of adhesive restorations happens more often due to insufficient sealing of cavity margins, with subsequent discoloration, rather than complete loss of retention $\square{ }^{2223} \square$. Results from the present study showed that the degree of coversion of tested adhesive agents that incorporated with FG nanoparticles and the pure adhesives are not change significantly. Secondary caries is one of the most common reasons which are responsible for the limited longevity of indirect restoration $\square^{\mathbf{2 3}} \square$.In previous studies, the antibacterial function of graphene is believed to becaused by both physical and chemical effects. The most important mechanism is the bacteria cell membrane destruction by the sharp edges of graphene nanosheets in suspensions $\square^{2} \square \square$. The chemical effect is primarily oxidative stress created by reactive oxygen species (ROS) $\square^{2} \square \square$ and extraction of phospholipids from the cell membrane. A study found that graphene layer reduces the attachment of microbes $\square^{2} \square \square$. Recently; researchers found that graphene oxide nanosheets would be an effective antibacterial material against dentalpathogens $\square^{2} \square \square$. Our research showed that the antibacterial behavior of the conventional adhesives could be enhanced by FG and the effect is more obvious for specimens with higher FG. Similar to graphene, the physical damage toward bacteria by sharp edges and chemical effect may also be responsible forits antibacterial property.

\section{Conclusion}

In addition, adhesive/FG composites showed superior performance of killing $S$. mutans and Lactobacilli bacteria. The presented work shows FG has potential to improve antibacterial properties and degree of conversion of adhesive agents.

Ethical Clearance: The Research Ethical Committee at scientific research by ethical approval of both environmental and health and higher education and scientific research ministries in Iraq.

Conflict of Interest: The authors declare that they have no conflict of interest.

Funding: Self-funding 


\section{References}

1. Tezvergil-Mutluay A, Mutluay M, SeseogullariDirihan R, Agee KA, Key WO, Scheffel DL. The Effect of phosphoric acid the degradation of human dentin matrix. J Dent Res. 2013; 92:87-91

2. Eliseu A. Münchow, Marco C. Bottino. Recent Advances in Adhesive Bonding: The Role of Biomolecules, Nanocompounds, and Bonding Strategies in Enhancing Resin Bonding to Dental Substrates.Curr Oral Health Rep. 2017; 23:211-22

3. Barcellos DC, Fonseca BM, Pucci CR, Cavalcanti B, Persici Ede S, Goncalves SE. Zn-doped etchand-rinse model dentin adhesives: Dentin bond integrity, biocompatibility, and properties. Dent Mater. 2016; 32:940-50.

4. DeMunck J, Van Landuyt K, PeumansM, Poitevin A, Lambrechts P, Braem M. A critical review of the durability of adhesion to tooth tissue: method and results. J Dent Res. 2005; 84:118-32.

5. Carvalho C, Fernandes FP, Freitas Vda P, Franca FM, Basting RT, Turssi CP. Effect of green tea extract on bonding durability of an etch-and-rinse adhesive system to caries-affected dentin.J Appl Oral Sci. 2016;24:211-7.

6. Novoselov KS, Fal'ko VI, Colombo L, Gellert PR, Schwab MG, Kim K. A roadmap for graphene. Nature2012; 490(7419):192-200.

7. Wang JJ, Wang F, Li JM, Sun Q, Jia Y. Theoretical study ofsuperlow friction between two singleside hydrogenatedgraphene sheets. Tribol Lett 2012;48(2):255-61.

8. Nair RR, Ren W, Jalil R, Riaz I, Kravets VG, Britnell L. Fluorographene: a two-dimensional counterpart of Teflon. Small 2010;6(24):2877-84.

9. Zhang SC, Kern M. The role of host-derived dentinal matrix metalloproteinases in reducing dentin bonding of resin adhesives. Int J Oral Sci. 2006; 1:163-76.

10. Baradaran S, Moghaddam E, Basirun WJ, MehraliM, Sookhakian M, Hamdi M. Mechanical properties andbiomedical applications of a nanotubehydroxyapatite-reduced graphene oxide composite. Carbon2013; 69:32-45.

11. Gao C, Liu T, Shuai C, Peng S. Enhancement mechanisms of graphene in nano-58S bioactive glass scaffold: mechanicaland biological performance. Sci Rep 2014; 4:4712.
12. Pham VT, Truong VK, Quinn MD, Notley SM, Guo Y, Baulin VA. Graphene induces formation of pores that killspherical and rod-shaped bacteria. ACS Nano 2015; 9:8458-67.

13. Tu Y, Lv M, Xiu P, Huynh T, Zhang M, Castelli $M$. Destructive extraction of phospholipids from Escherichia colimembranes by graphene nanosheets. Nat Nanotechnol 2013; 8:594-601.

14. Ji H, Sun H, Qu X. Antibacterial applications of graphene-based nanomaterials: recent achievements andchallenges. Adv Drug Deliv Rev 2016; 105(Pt B): 176-89.

15. Li Suna, b, Zhuanjun Yana, YouxinDuanb, JunyanZhangb, Bin Liua, Improvement of the mechanical, tribological and antibacterial properties of glass ionomer cementsby fluorinated graphene. Dent Mat J 2018, 23(5):484-9.

16. Deb S, Wang M, Tanner KE, Bonfield W. Hydroxyapatite-polyethylene composites: effect of grafting and surface treatment of hydroxyapatite. Journal of Materials Science 1996: Materials in Medicine 7(4), 191-193.

17. Kikutia SH, Saneblidze L, Mikelashvili V, Markhulia J, Tatarashvili R. Anew Method for the Synthesis of Nanoparticles for Biomedical Applications. Eur. Chem. Bull., 2015, 4(1), 33-36.

18. Silva GR, Silva NR, Sorces PV, Coater AR, Ferna AJ, Roare CJ. 2012; Influence of different load application devices on fracture resistance of restored premolars. Braz Dent J, 23(5): 484-9.

19. Gholam MQ, Hameed MR. Development and assessment of addition of Fluoroapatite or Calcium Fluoride to two types of adhesive (in vitro and vivo study). [Ph.D. thesis]. University of Baghdad, Department of Conservative and Esthetic Dentistry. 2018.

20. Fatemeh-Maleknejad Yazdi, Horieh Moosavi, Mohammad Atai, Mahsa Zeynali, Dentin bond strength and degree of conversion evaluation of experimental self-etch adhesive systems. J Clin Exp Dent. 2015;7(2):e243-9.

21. Racraddin, J. D. 1985. Media for isolationcultivation identification maintenance of medical bacteria, vol. 1, p. 275-284. Williams \& Wilkins, Baltimore, MD.

22. Van Landuyt K1, Snauwaert J, De Munck J, Peumans M, Yoshida Y, Poitevin A, Coutinho E, Suzuki K, Lambrechts P, Van Meerbeek B. Systematic review 
of thechemical composition of contemporary dental adhesives. Biomater 2007;26:3757-3785.

23. Van Meerbeek B, De Munck J, Yoshida Y, Inoue S, Vargas M, Vijay P, Van Landuyt K, Lambrechts P, Vanherle G. Buonocore Memorial Lecture. Adhesion to enamel and dentin: current status and future challenges. Oper Dent 2003;28: 215-35.
24. Czarnecka B, Nicholson JW. The biocompatibility of resin-modified glass-ionomer cements for dentistry. Dent Mater 2008; 24:1702-8.

25. Moreau JL, Xu HH. Fluoride releasing restorative materials: effects of $\mathrm{pH}$ on mechanical properties and ion release. Dent Mater 2010; 26:227-35. 